\title{
Antibacterial and antioxidant activities of phenolic compounds extracted from autumn fruits by-products
}

Vanessa Silva ${ }^{1-4 *}$, Apolo Martins ${ }^{1}$, Adriana Silva ${ }^{1-4}$, Alfredo Aires ${ }^{5}$, Rosa Carvalho ${ }^{6}$, Virgílio Falco ${ }^{7}$, Gillberto Igrejas ${ }^{2-4}$, Patrícia Poeta ${ }^{1,4}$

${ }^{1}$ Microbiology and Antibiotic Resistance Team (MicroART), Department of Veterinary Sciences, University of Trás-os-Montes and Alto Douro (UTAD), 5000-801 Vila Real, Portugal;

${ }^{2}$ Department of Genetics and Biotechnology, University of Trás-os-Montes and Alto Douro,

5000-801 Vila Real, Portugal;

${ }^{3}$ Functional Genomics and Proteomics Unit, University of Trás-os-Montes and Alto Douro (UTAD), 5000-801 Vila Real, Portugal;

${ }^{4}$ Associated Laboratory for Green Chemistry (LAQV-REQUIMTE), University NOVA of Lisboa, Lisboa, 2829-516 Caparica, Portugal;

${ }^{5}$ Centre for the Research and Technology of Agro-Environmental and Biological Sciences (CITAB), University of Trás-os-Montes and Alto Douro (UTAD), Vila Real,

Portugal;

${ }^{6}$ Department of Agronomy, School of Agrarian and Veterinary Sciences, University of Trás-os-Montes e Alto Douro (UTAD), Vila Real, Portugal.

${ }^{7}$ Chemistry Research Centre (CQ-VR), University of Trás-os-Montes and Alto Douro (UTAD), 5000-801 Vila Real, Portugal.

*vanessasilva@utad.pt

\section{Introduction}

In the last few decades there has been a dramatic increase in antibiotic resistant bacteria, particularly methicillinresistant Staphylococcus aureus (MRSA), that not respond to conventional treatments. In this study, the antioxidant and antibacterial activities of phenolic compounds extracted from by-products resulting from the autumn fruit industry were evaluated.

\section{Material and Methods}

Extraction of phenolic compounds from pomegranate, quince, loquat and persimmon industry by-products was performed using a mixture of ethanol/water (80:20). Antimicrobial susceptibility was tested against 10 MRSA strains: 5 MRSA isolated from human diabetic foot ulcers, 3 from healthy wild hares and 2 from consumption rabbits. Minimum inhibitory concentrations were determined by disc diffusion method using different concentrations of extracts $(100,75,50,25$ and $10 \mathrm{mg} / \mathrm{mL})$. Antioxidant activity was performed using the DPPH, CUPRAC and FRAP methods. Each determination was performed in triplicate.

\section{Results}

Most of the extracts had an inhibitory effect on the growth of MRSA strains from all origins except for quince seed and peel extracts and pomegranate seed extracts. Pomegranate peel extracts had the highest inhibitory effect on all MRSA strains presenting halos with diameter around $18 \mathrm{~mm}$ followed by persimmon calyx extracts (Table 1 ). Regarding the antioxidant activity, the extracts with the highest antioxidant power were pomegranate leaf extract, followed by persimmon calyx extracts, quince leaf and loquat flower.

\begin{tabular}{|c|c|c|c|c|c|c|c|c|c|c|c|}
\hline \multirow[t]{2}{*}{ Fruit } & \multirow{2}{*}{$\begin{array}{c}\text { Fruit } \\
\text { component }\end{array}$} & \multicolumn{10}{|c|}{ Inhbition zone (mm) } \\
\hline & & strain 1 & strain 2 & strain 3 & strain 4 & strain 5 & strain 6 & strain 7 & strain 8 & strain 9 & strain 10 \\
\hline \multirow{3}{*}{ pomegranate } & leaf & 12 & 17 & 12 & 13 & 15 & 17 & 12 & 15 & 14 & 12 \\
\hline & peel & 18 & 17 & 17 & 18 & 19 & 15 & 20 & 19 & 19 & 17 \\
\hline & seed & 7 & 0 & 0 & 0 & 0 & 0 & 0 & 0 & 0 & 0 \\
\hline \multirow{3}{*}{ quince } & leaf & 10 & 12 & 10 & 10 & 9 & 14 & 13 & 12 & 14 & 10 \\
\hline & peel & 0 & 8 & 0 & 0 & 0 & 10 & 11 & 9 & 0 & 0 \\
\hline & seed & 0 & 0 & 0 & 0 & 0 & 0 & 0 & 0 & 0 & 0 \\
\hline \multirow{4}{*}{ persimmon } & calyx & 12 & 16 & 11 & 15 & 17 & 17 & 16 & 16 & 14 & 12 \\
\hline & leaf & 10 & 14 & 10 & 11 & 12 & 16 & 15 & 12 & 16 & 11 \\
\hline & peel & 0 & 8 & 7 & 0 & 0 & 10 & 10 & 9 & 0 & 0 \\
\hline & seed & 10 & 15 & 10 & 10 & 10 & - & - & - & - & - \\
\hline \multirow{2}{*}{ loquat } & Leaf & 10 & 11 & 11 & 12 & 9 & 13 & 14 & 14 & 13 & 10 \\
\hline & flower & 8 & 11 & 9 & 9 & 11 & 12 & 11 & 11 & 10 & 10 \\
\hline
\end{tabular}

\section{Conclusions}

Extracts from by-products of several autumn fruits revealed a high antibacterial and antioxidant activities. These compounds may be used as antibiotic substitutes or adjuvants to combat antibiotic resistant bacteria or in food industry as food preservers.

\section{Acknowledgements}

This work was supported by the Associate Laboratory for Green Chemistry - LAQV which is financed by nationa funds from FCT/MCTES (UIDB/50006/2020). Vanessa Silva is grateful to FCT (Fundação para a Ciência e a Tecnologia) for financial support through PhD grant SFRH/BD/137947/2018. 